\title{
Asymptotic Freedom: A Possible Physical Explanation
}

\author{
Antonio PUCCINI \\ Neurophysiologist of Health Ministry, Naples - Italy \\ antonio.puccini.4rr1@na.omceo.it, antonio_puccini@libero.it
}

\begin{abstract}
The Quarks asymptotic freedom phenomenon is characterized by a certain movement independence of the quarks(Qs) but only for very short distances. This derives from the fact that when Qs are very close together, the Strong Interaction(SI) almost completely loses its strength. Why?

It could be assumed that this is a consequence of the shielding and masking effects in their turn supported by the congruous and elegant mathematical formalism of the Quantum Chromo-Dynamics, sufficient on its own to explain the phenomenon.

On the other hand we wonder: what is the objective, physical, concrete reality that underlies it? What is the exact physical mechanism for which the SI strength is not homogeneous throughout its action radius? Or: how can Qs, alone, without any help, have enough strength and power to get rid of the extremely intense grip exercised by the SI?

Unless there is the intervention of some other phenomena, currently unknown, completely unrelated to the SI. Then, one might wonder: is it possible that there is something between the Qs, which at first goes unnoticed, but when the particles gather each other excessively this something begins to be felt, showing a clear and energetic repulsive action? But if so, what is it?

We believe that in the asymptotic freedom it is not the SI to loose strength, but it is overwhelmed by another force, a Repulsive Force, quite distinct from it, and that, in certain peculiar circumstances, proves to be even more powerful than the SI itself.
\end{abstract}

Keywords: Quarks(Qs); Electromagnetic Radiation(EMR); Photon(P); Dark Energy(DE).

1. Introduction

2. Discussion

2.1 Levy Interaction

2.2 Possible Physical cause of the Asymptotic Freedom

2.2.1 Identikit of Intra-Nuclear and Intra-Nucleonic Repulsive Force

2.3 Vacuum Energy

2.4 Dark Energy (DE)

2.5 Contexts and Operating Modes of DE

2.5.1 Exterminated Sidereal Spaces

2.5.2 Big Bang

2.5.3 Stellar Cores

2.5.4 Inflationary Phase

2.5.5 Intra-Atomic Spaces

2.5.6 Intra-Nuclear Spaces

2.5.7 Intra-Nucleonic Spaces

3.Conclusions References 


\section{Introduction}

As is well-known, the 3 quarks ( $Q_{s}$ ) confined within the nucleon have a certain autonomy of movement. This independence of the Qs at very short distances, within a very small area, defined asymptotic freedom, has been described simultaneously by Gross and Wilczek[1][2] and by Politzer[3]. The calculation of the A.A. concerns the determination of the transferred high momentum trend, $q^{2}$, of the actual constant of a non-Abelian theory as the Quantum Chromo Dynamics (QCD). The result shows that the actual constant, $g^{2}$, asymptotically tends to zero as $q^{2}$ increases:

$$
g\left(q^{2}\right) \approx \frac{c}{\log (q)^{2}} \rightarrow 0\left(q^{2} \rightarrow \infty\right)
$$

The interactions between $Q_{s,}$ extremely intense at the energies and moment transferred from the order of $q^{2} \sim 1 \mathrm{GeV}^{2}$, decrease in intensity and tend asymptotically to a situation in which the $\mathrm{Q}_{\mathrm{s}}$ behave as if they were free: a property admirably defined asymptotic freedom of the Strong Interactions(SIs). The result, at the time completely unexpected, justifies the scale relations in the cross sections observed at the end of the 1960s and in the early 1970s in the electron-proton and neutrino-proton diffusion processes with high transfer momentum[4].

In this respect, Maiani specifies that the scale laws, already identified by Bjorken[5], are interpreted by Feynman as indicative of the fact that - in the diffusion of the electron or neutrino - the $Q_{s}$ behave as if they were free from interaction. The experimentation of the electrons and neutrinos diffusion processes, at large transferred momenta (deep inelastic scattering), has led to the conclusion that the proton (and every other hadron) participates in the processes with great transferred momentum. As if it were made of an incoherent whole of gluons $\left(G_{s}\right)$, $Q s$ and anti- $Q_{s}\left(Q_{s}\right)$ of the different flavors, each characterized by a function, $P i(\mathrm{x})$, which gives the probability of finding in the proton a $\mathrm{Q}$ of a given type(up, down, strange...) with a fraction $\mathrm{x}$ of the momentum of the proton itself [4]. The Pi(x) are called functions structure of the proton.

The logarithmic approach to asymptotic freedom, Eq.(1), causes that the scale relations are affected by logarithmic corrections, which have been accurately calculated by different authors, including Altarelli, Parisi [6] and Dokshitzer [7], and subsequently compared with experimental data, at energies gradually increasing. Quigg points out: "We can think of a hadron as a bubble, in which $Q_{s}$ are imprisoned. Inside the bubble $Q_{s}$ move freely, but they cannot escape. It is believed, that is, that the Strong Interaction(SI) undergoes a shielding action, since the virtual color charges (represented essentially by $Q$ Q pairs) fill the intra-hadron vacuum. In other words, a $Q$, which also carries a color charge, attracts the opposite colors, thus surrounding itself with a shield, which reduces its effective charge with distance. However, this shielding effect is counteracted by the so-called masking effect, whereby a $Q$ radiates and continuously reabsorbs $G_{s}$ which take its color charge to considerable distances and change its color. The charge takes its complete action only outside the space it occupies. Therefore the masking increases the force acting on a true $Q$ as it moves away from the $1^{\circ} Q$ towards the border of the region with color charge. The net result of shielding and masking is that at short distances the $\mathrm{SI}$, based on the color charge, is weaker, while at longer distances it is more intense"[8]. The QCD equations [9] state that $Q_{s}$ are perennially confined and asymptotically free.

QCD gives us a model of SIs described by a non-Abelian gauge Lagrangian, very similar to that describing the electromagnetic field:

$$
L_{Q C D}=-\frac{1}{4} F_{\mu \nu}^{(a)} F^{(a) \mu \nu}+i \sum_{q} \bar{\psi}_{q}^{i} \gamma^{\mu}\left(D_{\mu}\right)_{i j} \psi_{q}^{j}-\sum_{q} m_{q} \bar{\psi}_{q}^{i} \psi_{q i}
$$


where $F_{\mu \nu}{ }^{(a)}$ is the is the non-Abelian gauge-covariant curl. It represents the tensor (curl) describing the intensity of the strong field (it is considered an anti-symmetric tensor). The $\Psi^{i}{ }_{q}$ fields are the Dirac $\Psi$ and anti $-\Psi(\Psi)$ spinors, indicating respectively $Q$ and $\bar{Q}$ color multiplets, of which $i$ characterizes the color (the new parameter introduced with $\mathrm{QCD}$ ) and ${ }_{q}$ the flower; $Y^{\mu}$ are Dirac matrices; $m$ is the Qmass matrix; the symbol $\Sigma$ expresses all the possible spatial positions occupied by the particles, while $\left(D_{\mu}\right)_{i j}$ is the gauge covariant derivative:

$$
\begin{gathered}
F_{\mu \nu}^{(a)}=\partial_{\mu} A_{\nu}^{a}-\partial_{\nu} A_{\mu}^{a}-g_{s} f_{a b c} A_{\mu}^{b} A_{\nu}^{c} \\
\left(D_{\mu}\right)_{i j}=\delta_{i j} \partial_{\mu}+i g_{s} \sum_{a} \frac{\lambda_{i j}^{a}}{2} A_{\mu}^{a}
\end{gathered}
$$

and where $g_{s}$ is the coupling constant of the $\mathrm{SI}, f_{a b c}$ are the group structure constants of the group of symmetry, called $S U(3)$. The coefficients $\lambda^{a}{ }_{i j}$ are coupling constants in matrix form, called Gell-Mann matrices. These matrices obey to the following commutation relation:

$$
\left[\lambda^{a}, \lambda^{b}\right]=i f^{a b c} \lambda^{c}
$$

To this purpose, it seems useful to precise that the strong field, placed inside the hadrons, is a quantum field capable of preserving a local gauge symmetry, which persists even after a partial transformation of the field itself. QCD gives us a model of SI described by a non-Abelian gauge Lagrangian $(L)$-see Eq.(2)which describes the interactions between $Q_{s}$ and $G_{s}[9][10][11][12]$.

\section{Discussion}

As we all know, leptons do not carry any color, that is they have a null color charge, thus they are not sensitive to SI. "The color is the label of an exact representation $S U(3)$ of the states"[13]:

$$
r=\left(\begin{array}{l}
\mathbf{1} \\
\mathbf{0} \\
\mathbf{0}
\end{array}\right) g=\left(\begin{array}{l}
\mathbf{0} \\
\mathbf{1} \\
\mathbf{0}
\end{array}\right) \quad b=\left(\begin{array}{l}
\mathbf{0} \\
\mathbf{0} \\
\mathbf{1}
\end{array}\right)
$$

Let's consider, for istance, that a red $Q$ scatters on a green $Q$ : what happens is that the color is exchanged and always kept. As for the Gs

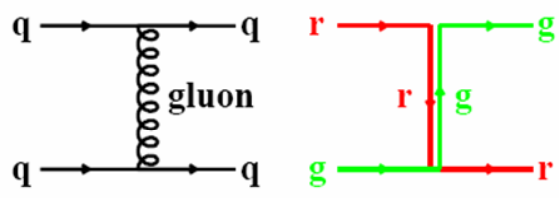

we expect 9 Gs (3 colours for 3 anti-colours):

$$
\begin{gathered}
r \bar{b}, r \bar{g}, g \bar{r}, g \bar{b}, b \bar{g}, b \bar{r} \\
r \bar{r}, g \bar{g}, b \bar{b}
\end{gathered}
$$

However, $\mathrm{Gs}$ are orthogonal combinations of the 9 states. The combination:

$$
\frac{1}{\sqrt{3}}(r \bar{r}+g \bar{g}+b \bar{b})
$$


s colourless (we get it as a color singlet), so it is not sensitive to $\mathrm{SI}$ : we remain with $8 \mathrm{Gs}_{\mathrm{s}}$ [13]. Along with QCD a gauge theory is applied with a symmetry group $S U(3)$, in which $Q_{s}$ are triplets belonging to this group, in which also Isospin and the strangeness of the considered particles are considered, so that hypothesis, according to Gell-Mann, was also called Eightfold Way, since it includes 8 independent

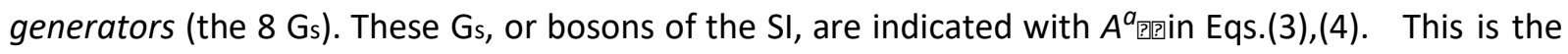
representation of Eightfold Way:

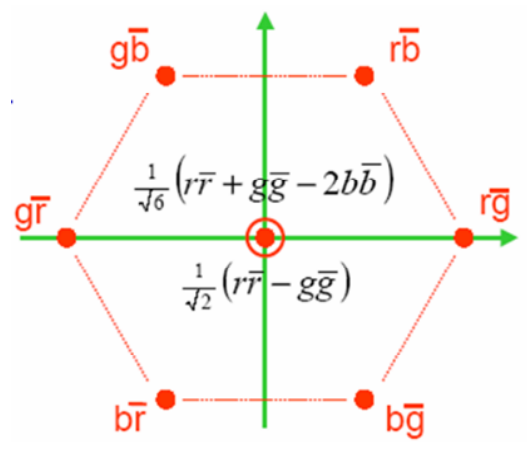

We now know that even the color is confined and that only the color singlet states are able to exist as free particles. A massless G, color singlet, would give rise to a long range SI [13]. However, these forces have never been observed, so we have to admit that there are only 8 physical $\mathrm{G}_{\mathrm{s}}$, color carriers, so they are permanently confined!

Moreover, since also Qs carry color, Qs are permanently confined too! Therefore, free particles can be made as follows[13]:

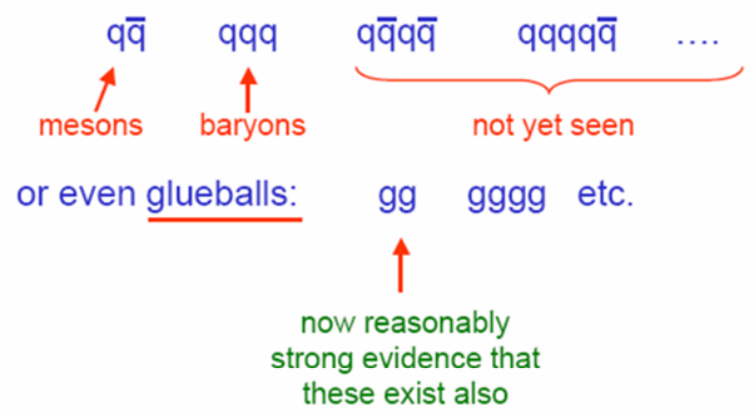

Thus, as can be seen from Mathematics, the asymptotic freedom is characterized by a certain movement independence of Qs, but only for very short distances. This derives from the fact that when Qs are very close together, the SI almost completely loses its strength. Why? It could be assumed that this is a consequence of the shielding and masking effects, previously exposed, in their

turn supported by the very valid mathematical formalism of the QCD, evidenced by the Eqs. (1),(2). These are all satisfactory explanations. Yet it is as if something is missing.

In short, on one hand we have the asymptotic freedom phenomenon, illustrated by a congruous and elegant mathematical formalism, sufficient on its own to explain the phenomenon; on the other hand we wonder: what is the objective, physical, concrete reality that underlies it?

What is the exact physical mechanism for which the SI strength is not homogeneous throughout its action radius? Or: how can $Q_{s}$, alone, without any help, have enough strength and power to get rid of the extremely intense grip exercised by the SI? 
One could reply: it is due to $Q_{s}$ which, if too close to each other, cannot be further gathered. Or, one could answer: it is an intrinsic characteristic of the $\mathrm{SI}$, so that as the inter-nucleonic distance, the spaces in which the SI operates, decreases, likewise the strength of the SI decreases.

And yet, they are unsatisfactory answers: they seem more like a description of the asymptotic freedom effect, rather than an explanation of its cause. Otherwise, a more elaborate explanation could consist in the decrease of the so-called anti-screening effect. As it is known, in fact, each $\mathrm{G}$ carries both a color charge and an anti-color charge. The net effect of virtual $\mathrm{Gs}_{\text {s }}$ polarization in the vacuum is not to hide the field, but to increase it and to influence its color: anti-screening effect. Thus, approaching further a $Q$, the anti-screening effect of the surrounding virtual $G_{s}$ decreases, so the contribution of this effect can determine a weakening of the effective charge as the distance decreases. This description is persuasive and appropriate, however it does not seem to provide us with an exhaustive explanation of this peculiar behavior of the SI, since it does not outline and does not clarify what the physical cause is.

Unless there is the intervention of some other phenomenon, currently unknown, completely unrelated to the SI. As it is known, in fact, from the analysis of the $Q_{s}$ Confinement, it clearly emerges that the main limit of the $\mathrm{SI}$ is its action radius, since this nuclear force won't be able to take another way, having to immediately remedy the energy debt. Thus, even if the too narrow space between contiguous particles ( $Q_{s}$ or nucleons) should more or less casually re-enter the field of action of the $\mathrm{Sl}$, with great surprise we will see that the strength of the $\mathrm{SI}$ is overwhelmed, since it fails to further gather the particles to each other.

This seems to us to be the keystone; this is the real enigma! At this point, one might wonder: is it possible that there is something between the nucleons, or between the $Q_{s}$, which at first goes unnoticed, but when the particles gather each other excessively this something begins to be felt, showing a clear and energetic repulsive action? But if so, what is it?

And yet, the existence of an intra-nuclear force, operating among nucleons, had been hypothesized in 1950s.

\subsection{Levy Interaction}

At this regard, we would like to quote the so-called N-N Force or Levy Interaction[14]. It is a repulsive force, which prevents the excessive approach of two nucleons, indicated as $\mathrm{N}-\mathrm{N}$. Indeed, as known, the particles cannot approach each other beyond a given distance $\left(d_{0}\right)$, below which a repulsive force appears: Levy Interaction (LI). In this respect, Wigner and Eisenbud specify: "There is experimental evidence that the $\mathrm{SI}$ is repulsive at a distance very small among the nucleons. A particular potential, which was originally proposed on the bases of the meson theory of nuclear forces, and that gives a fairly good description of the systems with two bodies, it is the $L I$. This force is intensely repulsive at very short distances. Between two nucleons, $L /$ is strongly repulsive from distances $\left(d_{0}\right)$ "[15] equal to:

$$
d_{0}<0.55 \cdot 10^{-13}[\mathrm{~cm}]
$$

A very similar phenomenon occurs within the nucleons, between Qs. Thus, in line with Gross, Wilczek[1],[2] and Politzer [3], we cannot exclude the possible existence of a repulsive effect, analogous to the one hypothesized for the $L I$ (likely operating within the intra-nuclear space, among nucleons too close together) even in the intra-nucleonic area. It starts being detected when the particles present in this intra-nucleonic field, i.e. $Q_{s}$, subjected to the very intense attractive action exerted by the intra-nucleonic $\mathrm{SI}$, or Color Force(CF), come too close till prevailing over the $\mathrm{SI}$, and therefore rejecting. 
Antonio PUCCINI; Asymptotic Freedom: A Possible Physical Explanation, Advances in Image and Video Processing, Volume 7 No 6, December (2019); pp: 17-40

At this regard, Pacini points out: "Among the nucleons, regardless of their charge, there is a very powerful attractive force, the SI, which prevails on the Coulomb Force (repulsive between protons) when the distance between the two interacting nucleons is $\leq 10^{-13}[\mathrm{~cm}]$, that is 1 fermi.

But by compressing the nucleons enough, the force becomes repulsive again!

Indeed, the intervention of this force places a limit on the further reciprocal approach of the nucleons, limit corresponding to 0.30 fermi, beyond which there is a saturation barrier"[16]:

$$
R f \approx \leq 0.30 \cdot 10^{-13}[\mathrm{~cm}]
$$

where $R f$ indicates a Repulsive force, whose radius of action appears to be superimposable (just a little shorter) to LI's. Similarly to Levy, also what Pacini described, seems possible to occur, with the same modalities, within the nucleons, among the Qs, according to the description of the asymptotic freedom phenomenon elaborated by Gross, Wilczek and Politzer [1],[2],[3].

Both Levy's description, and Gross-Wilczek-Politzer's, use an elegant mathematical formalism, which shows the disappearance of the SI when the nucleons, or the Qs, are too close together.

This represents the crucial point, in our opinion, to try to deepen this peculiar phenomenon, able to override, eventually, the most powerful known force: right for that called SI!

However, we feel that the Mathematics, which describes asymptotic freedom so brilliantly, does not fully clarify the real, objective, physical cause which can generate such phenomenon, but it just shows us the effects.

\subsection{Possible Physical Cause of the Asymptotic Freedom}

We believe that in the $Q_{s}$ asymptotic freedom it is not the SI to loose strength, but it is overwhelmed by another force, a Repulsive Force $(R f)$, quite distinct from it, and that, in certain peculiar circumstances, proves to be even more powerful than the SI itself.

We reiterate, in our opinion this is the heart of the asymptotic freedom phenomenon.

In reference to this context, Pacini adds: "But there is more: to be convinced of this $R f$, which acts as 'repulsive', as for trains, between the two particles, we should think that without it, the atomic nucleus would not hold up and would tend to shrink more and more"[16].

This statement gives a primary and absolute value to this $R f$ : without its very strong repulsive action, he world would not be as it is!

Obviously, one wonders: where does this $R f$ come from? What is it?

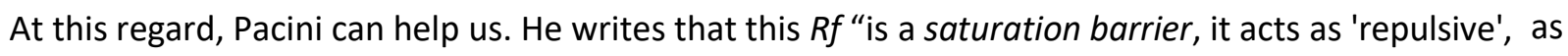
for the train bumper, between the two particles"[16], nucleons or Qs as they are.

But this requires another question, which is fundamental in our opinion: what is the nature of this saturation barrier, acting as a $R f$ ? Let's try to build its the profile.

\section{2 2 Identikit of Intra-Nuclear and Intra-Nucleonic Repulsive Force}

Firstly, according to Pacini this $R f$ manifests its presence both when the inter-nucleonic space shrinks excessively (so it is present inside the atomic nuclei, or Heisemberg Isospin field[17]), and when the $G_{s}$ are too close together (so it is also present within the intra-nucleonic space). In this respect, we read: "This mysterious repulsive energy antigravity, or $5^{\text {th }}$ Force, should also act against the $\mathrm{G}_{\mathrm{s}}$, thus succeeding in overriding the $\mathrm{SI}$ when the $\mathrm{Q}_{\mathrm{s}}$ tend to get too close to each other, that is when they 
almost touch each other, but not really: that is there is always some space between the Qs. The space is apparently empty, but actually it is occupied by the 'thickness' of the $5^{\text {th }}$ Force" [18]. Then, the profile of our intra-atomic $R f$ is enriched: it is associated with the very famous thus, the mysterious, anti-gravity, repulsive energy, or $5^{\text {th }}$ Force, Quintessence, better known as Dark Energy (DE).

At this point, one may object: it is wrong! The DE is not confined within atoms. DE was discovered in the infinite intergalactic spaces, identified with that energy of repulsive, antigravity action, responsible for the accelerated expansion of the Universe [19] [20].

It's right. However, DE was considered to be, primarily, in close correlation with the vacuum energy. Let's try to better understand what "vacuum" and vacuum energy mean.

\subsection{Vacuum Energy}

In the Universe there is another mysterious force, never directly observed, "called vacuum energy or negative pressure, or simply strange energy: this force is opposed to the force of attraction of gravity, accelerating the expansion of the Universe"[21]. This antigravity counter-pressure, exerted by the energy of vacuum, also for many others physicists, is equivalent to the DE, which Hack points to as a 5th Fundamental Force, corresponding to cosmological density[22]. Hack adds: "Let's imagine to consider a region of space and take away all matter, radiation and every other kind of substance. The resulting state is called vacuum, which is something different from null. The vacuum has the lowest energy of any other state, but not necessarily zero. According to Relativity Theory, every form of energy influences the gravitational field, and therefore the energy of the vacuum becomes an important ingredient. It is believed that the vacuum is the same throughout the universe, and consequently the energy density of the vacuum is called cosmological constant $(\Lambda)$. While matter can be thickened or dispersed during the evolution of the universe, $\Lambda$ is a property of the space-time"[22]. Hawking says: "Besides matter, the universe can contain energy of the vacuum, the energy present also in an apparently empty space"[23].

According to Chandrasekhar, indeed, in the description of physic systems, vacuum is considered as the minimum energy state, or Zero Point Energy (ZPE)[24][25], which only in some cases corresponds to the almost total absence of particles or waves. "It was thought that the interstellar and inter-galactic spaces were expanses of vacuum, but then with the theory of quantum fields(QFT) it was stated that space is never really empty, but is pervaded by quantum fields present everywhere: the various particles are, in fact, excited states of these fields. Space appears empty when the fields are at the lowest energy level, whereas space comes alive with visible matter and energy when the fields are excited. Wheeler said: empty space is not empty, but it is the kingdom of the richest and most surprising Physics. For Hawking, DE is the central problem of Physics"[26].

Barrow writes: "The quantum revolution has shown us why the old concept of vacuum as an empty box was unsustainable. From then on, the vacuum was simply the state that remained when everything that could be removed from the box had been removed. This state was by no means the absence of anything: it was only the lowest possible energy state. There was always something remaining: an energy of emptiness that permeated every fiber of the universe. It is never possible to achieve a perfect vacuum. Any small perturbation or attempt to intervene on the vacuum would increase its energy. The omnipresent, un-eliminable vacuum energy was revealed and proved to have a tangible physical presence. Einstein showed that the universe could contain a mysterious form of vacuum energy. The Uncertainty Principle (HUP) and quantum theory have revolutionized the concept of vacuum. Saying that in a box there are no particles, that it is completely free from any mass and energy, is in contrast to the HUP, as it assumes to have complete information on the motion at any point and on the energy 
Antonio PUCCINI; Asymptotic Freedom: A Possible Physical Explanation, Advances in Image and Video Processing, Volume 7 No 6, December (2019); pp: 17-40

of the system at a given instant of time. The entity of the uncertainty is precisely the so-called ZPE"[27].

Still with regard to the vacuum energy, it should be specified that "in classical physics the vacuum is identifiable with the absence of energy. In contrast, in the Quantum Mechanics, the HUP prevents a measure of the vacuum state energy from giving exactly a zero value. Because of the HUP the number of particles contained in the vacuum state cannot be null, but it is forced to undergo random fluctuations: the quantum vacuum must therefore be imagined as a dynamic state, rich in all the particles -called virtual (that is, of very short life)- which are produced due to unavoidable quantum fluctuations"[26]. Barrow specifies: "The quantum vacuum can be conceived as a sea made up of elementary particles of all types and their antiparticles, which appear and disappear continuously. We focus our attention only on the Electro-Magnetic Interactions: there will be a great ferment of electrons and positrons. Electron-positron pairs materialize from the quantum vacuum and then immediately they annihilate each other, disappearing. If the electron and positron have mass ( $\mathrm{m}$ ), Einstein formula $\left(E=m c^{2}\right)$ tells us that their "creation" requires an energy $(E)$ of $2 \cdot m^{2}$, which must be borrowed from the vacuum"[27]. This implies that the vacuum, the quantum vacuum, contains a fair amount of energy, since it is able to lend it!

This is a crucial point, in our opinion. That is, it is agreed that the quantum vacuum teems with energy, including DE. Furthermore, among the known energies, as reported by numerous A.A., that widely present in the vacuum, or quantum vacuum, is the Electromagnetic Radiation (EMR), as to say: photons $\left(P_{s}\right)$. Further, the EMR is qualified to be part of the quantum vacuum energy; EMR is just a quantum energy: in fact, it is conveyed by light's quanta!

At this regard, Randall comforts us: "The intra-atomic space swarms of EMR" [28]. Hack writes: "The primordial plasma was subject to two opposing forces:1) Gravity and 2) Radiation Pressure (or Photonic Pressure). The former tends to compress the gas until the Photonic Pressure reverses its motion, producing elastic oscillations. Since compression heats the plasma, it results in the warmer and colder regions observable in the cosmic microwave background(CMB), shown by the presence of two peaks. Since both the baryonic matter and the Dark Matter are subject to the Gravity Interaction(GI), while only the baryonic matter is subject to radiation pressure, it is possible to determine from the properties of the peaks that the baryon matter is $\approx 4 \%$ of the critical density and the Dark Matter $\approx 23 \%$. On the other hand, the fact that the universe is flat means that the critical density must be equal to 1 . It follows that the remaining $73 \%$ consists of energy density"[22]: the socalled DE. "But which energy?"[22] the Author is asked. "It is supposed to be an energy, discovered in recent years, called vacuum energy, which causes an accelerated expansion of space, while it was always expected that the Gl would decelerate the expansion. This means that there is a force acting against gravity. The energy that causes the acceleration of the expansion is the vacuum energy and, since energy and matter are equivalent, it probably provides that $73 \%$ density necessary to bring the density of the universe to the critical value, compatible with the observations that establish that the universe is flat "[22].

Briefly, the laws of Quantum Mechanics tell us that the apparently empty space is full of particles of every kind, which appear and immediately disappear, generating a repulsive force very similar to that which would be generated by the cosmological constant $(\Lambda)$, as to say: DE. 


\subsection{Dark Energy}

The Dark Energy(DE) is a sort of intrinsic energy of space. DE is a form of invisible energy, unknown and repulsive, that does not dilute with the expansion of the Universe, and does not interact with ordinary matter. DE is a hypothetical form of energy that exerts a negative, repulsive pressure, behaving like the opposite of gravity. "DE is a field of energy, or a property of unknown space, capable of opposing gravity"[29]. We do not know the origin of the DE.

We read from CERN: "DE makes up approximately $68 \%$ of the universe and appears to be associated with the vacuum in space. It is distributed evenly throughout the universe, not only in space but also in time - in other words, its effect is not diluted as the universe expands. The even distribution means that DE does not have any local gravitational effects, but rather a global effect on the universe as a whole. This leads to a repulsive force, which tends to accelerate the expansion of the universe. The rate of expansion and its acceleration can be measured by observations based on the Hubble law. These measurements, together with other scientific data, have confirmed the existence of DE and provide an estimate of just how much of this mysterious substance exists"[30].

In the NASA captions we find: "DE is a truly bizarre form of matter, or perhaps a property of the vacuum itself, that is characterized by a large, negative pressure. $\mathrm{DE}$ is the only form of matter that can cause the expansion of the universe to accelerate, or speed up"[31].

Hence, among the various proposals put forward by physicists and cosmologists, in order to identify the DE, there are two hypotheses most followed: 1 ) The Cosmological Constant $(\Lambda)$, which represents a constant energy density that fills the whole space homogeneously. 2) Scalar Fields, such as Quintessence and modules, i.e. dynamic quantities: Quintessence is a dynamic field, which energy density can vary in time and space (the contributions of the Scalar Fields that are constant in space are usually included also in $\Lambda$ ). The $\Lambda$ can be formulated to be equivalent to the radiation of the empty space, or the vacuum energy. Scalar Fields that change in space can be difficult to distinguish from $\Lambda$, since change can be extremely slow.

\subsection{Contexts And Operating Modes Of DE}

Widespread opinion is that $\Lambda$ represents a force of repulsion among the masses, able to act only between huge masses and over very great distances. However we do not think it always works this way. We have clear evidence that the DE also acts on very short, intra-atomic distances, since it is considered to coincide with the energy of vacuum, "vacuum also present inside the atom and represented by the electromagnetic field"[21].

Shortly, we have various examples of probable operating contexts of the DE, often very different from each other, both in terms of the extent of the space in which it operates, and with regard to the intensity of the energy with which it operates, and with regard to the methods and times in which it carries out its action.

\subsubsection{Exterminated Sidereal Spaces}

The best known context in which the DE is supposed to carry out its repulsive action is represented by the exterminated sidereal spaces in which, with deep surprise, in 1998 an acceleration of the expansion of the Universe was found [19][20]. This acceleration has been attributed to a repulsive, anti-gravity action most likely carried out by a mysterious, elusive, impalpable form of energy, called precisely DE. In this case, in line with the calculations of Perlmutten team[20] the energy density of this repulsive force, or $\mathrm{DE}$, is $\sim 10^{-29} \mathrm{~g} / \mathrm{cm}^{3}$. 
Antonio PUCCINI; Asymptotic Freedom: A Possible Physical Explanation, Advances in Image and Video Processing, Volume 7 No 6, December (2019); pp: 17-40

We find very important emphasize that this repulsive force, or $\mathrm{DE}$, operating in the immense cosmic spaces, in our opinion it coincides with the repulsive force $(R f)$ reported by Levy, Eisenbud, Wigner and Pacini operating at intra-nuclear level. Moreover, despite its very small value, the DE arrives to represent as much as $68.3 \%$ of the entire mixture of mass-energy that permeates the cosmos[32],[33]. And 'interesting to note that, in this context, DE has carried out its action for exterminated distances and since the Big Bang.

\subsubsection{Big Bang}

Rovelli adds: "What happened before the Big Bang(BB)? In the Loop Theory, which combines Quantum Mechanics $(Q M)$ and General Relativity, based on the proposal by Martin Bojowald, who applies the Loop theory equations to cosmology, we come across a surprising result: the history of the Universe continues backwards over time and does not stop at the BB. It goes further back: the BB was a rebound (bounce) from a previous contraction (or Big Crunch). This 'bounce', says Bojowald, is due to the density of the contraction material, which when it becomes high comes into play the $Q M$ producing a kind of Repulsive Force (not entirely dissimilar to the repulsive force of quantum origin that prevents electrons from falling on the atomic nucleus) which bounces the contraction universe, thus giving rise to expansion, to the BB. In fact, the universe expands from a central region, from a very limited space, to very high density. Proof of this is the CMB which is spread throughout the universe and is a direct trace of the great initial warmth of when the cosmos was very compressed. Near the BB the matter is so dense, entering a region where the $Q M$ cannot be neglected "[34]. According to this concept, Ashtekar described with an elegant mathematical formalism that the quantum properties of space-time bring out something new: a Repulsive Force, which would have produced the rebound (bounce) of our universe, manifested with the $B B$, consequent to the violent Big Crunch of the previous universe [35].

Hence, the BB represents the oldest context of the anti-gravitational, repulsive action explained by the DE which, in our opinion, results completely identifiable with the Repulsive Force mentioned by Rovelli, Bojowald and Ashtekar. That is, the BB may be the effect of a bounce from a previous contraction (Big Crunch) [34][35]. Bounce due to the progressive increase in the density of the matter-energy in contraction, by an overwhelming Gravity Interaction(GI), such as to reach a compression and density limit, until the $Q M$ intervenes and triggers a real explosion.

In this circumstance the situation is completely reversed (compared to context 1): at the time of the BB the space in which the DE operates is not the entire Universe, but a very limited space, even less than a point according to Lemaitre. Also regarding the time we are at the antipodes. In the first example the $D E$ is operative from $\approx 13820$ thousand years, at the time of the $B B$ the action of the DE lasts only fractions of billionths of a second. Furthermore, the energy intensity of the DE shows abysmal differences: compared to the modest one of the first example (discussed in the $1^{\text {st }}$ context).

Based on our calculations, the energy with which the DE triggered the BB must have been far greater than that carried by the most energetic $\gamma$ rays[36].

\subsubsection{STELLAR CORES}

Another context in which the DE operates, is represented by a trial of strength that goes on uninterruptedly in the depths of the stellar cores between GI and DE. The gravity (GI) and the DE, represented, in this context, by the Radiation Pressure of the photons, can fight for a long time as it happens in the star's core[37],[38]. To this purpose, Feynman specifies: "When light is shining on a charge and it is oscilling in response to that charge, there is a driving Force in the direction of the 
light beam. This Force is called Radiation Pressure or Light Pressure (F). Let us determine how strong the Radiation Pressure is. Evidently it is that the light's force $(F)$ on a particle, in a magnetic

field $(B)$, is given by $F=q \mathbf{v} \cdot \boldsymbol{B}$, and it is at right angles both to the field and to velocity $(\mathbf{v}) ; q$ is the charge. Since everything is oscillating, it is the time average of this, $\langle F\rangle$. We know that the strength of the magnetic field is the same as the strength of the electric field $(\boldsymbol{E})$ divided by $c$ (the velocity of light in vacuum), so we need to find the average of the electric field, times the velocity, times the charge, times $1 / c$ :

$$
F=q \frac{\mathbf{v} E}{c}
$$

But the charge $q$ times the field $E$ is the electric force on a charge, and the force on the charge times the velocity is the work $d W / d t$ being done on the charge! Therefore the force, the Pushing Momentum, that is delivered per second by the light, is equal to $1 / c$ times the energy absorbed from the light per second! That is a general rule, since we did not say how strong the oscillator was, or whether some of the charges cancel out. In any circumstance where light is being absorbed, there is a Pressure. The momentum that the light delivers is always equal to the energy that is absorbed, divided by $c$ :

$$
F=\frac{\frac{d W}{d t}}{c}
$$

That light carries energy we already know. We now understand that it also carries momentum, and further, that the momentum carried is always $1 / c$ times the energy. The energy(E) of a light-particle is $h$ (the Planck's constant) times the frequency $(v)$ "'[39]:

$$
E=h v
$$

The $h$ value corresponds to $6.626 \cdot 10^{-27}$ [erg.s]; in its turn $v$ indicates the number of oscillations [Cs] in a second. Let's indicate with $n$ this number of oscillations $[c / s]$, i.e. $10^{n}[c / s]$. Thus, if we want to consider the Energy of a $P$, we should have:

$$
\begin{array}{ll}
E & =h \cdot v=h \cdot 10^{\mathrm{n}}[\mathrm{c} / \mathrm{s}] \\
\text { that is: } & E=6.626 \cdot 10^{-27}[\mathrm{erg} \cdot \mathrm{s}] \cdot 10^{\mathrm{n}}[\mathrm{c} / \mathrm{s}] \\
\text { hence: } & E=6.626 \cdot 10^{-27+\mathrm{n}}[\mathrm{erg}]
\end{array}
$$

This should be the Energy value of a P whose frequency is not specified[40].

From an authoritative source, we learn: "In ordinary stars such as our Sun, the inward force of gravity is balanced by the outward hydrodynamic pressure of the hot gasses and, to a lesser extent, by the radiation pressure of photons" [41].

Hence, the photons $\left(P_{s}\right)$ contribute to counter-balance the huge gravitational pressure which pushes from the outward external layers of the star to the internal layers. In order to perform this action, this compression, $\mathrm{Ps}_{\mathrm{s}}$ have to "base it on something", as though they had an equivalent mass [42],[43]

That is, it could be the equivalent mass of lots of billion of billion.. of $\mathrm{P}_{\mathrm{s}}$, which summed up may contribute, together with the "hydrodynamic pressure of the hot gases"[41], to prevent the Sun from collapsing or the collapse of the other stars, at least for a long time [44].

In other words, Ps exhibit "a mechanical effect"[45], realistically a mass effect acting as "counter pressure" to the considerable GI expressed by the remarkable gravitational mass, which inexorably pushes towards the inside of the star[46]. 


\subsubsection{Inflationary Phase}

As for the Inflationary Phase, Randall writes: "The measure of certain gravitational effects indicates the presence of something that is even more mysterious than Dark Matter: it is what is called DE. This DE that permeates the Universe is very similar to the energy that precipitated inflation, but today its density is much smaller than the energy that long ago presided over inflation"[28]: we pass, in fact, from $\gamma \mathrm{Ps}$ of an unimaginable energy to very weak microwaves. This is in perfect agreement with our hypothesis, both regarding inflation and DE: it is a very significant confirmation that DE can be constituted by Ps!

The concepts just reported by Randall, are in full agreement with what was proposed by Alan Guth. With his 'Inflationary theory', Guth hypothesized that a negative pressure field, similar in concept to $\mathrm{DE}$, could have led an Inflationary Phase in the primordial Universe[47]. Inflation postulates that a repulsive force, qualitatively similar to $\mathrm{DE}$, has caused a huge and exponential expansion of the Universe immediately after the Big Bang(BB). However, the inflation must absolutely have taken place at a much higher energy density than the energy density of the DE we observe today.

It has not been described if there is a relationship between DE and Inflation. However, in our opinion, the relation does exist: they are both conveyed by electro-magnetic radiation(EMR), but with extremely different energies, as we discussed in a Symposium held in Cambridge(Ma) [36].

To this purpose, it is necessary to consider the Einstein mass-energy equivalence principle[48]:

$$
E=m c^{2} \rightarrow m=\frac{E}{c^{2}}
$$

Thus, let's to insert the Energy(E) of the $P$ (as shown in Eq.13) in the Eq.(14):

$$
m_{\circ}=\frac{E}{c^{2}}=\frac{6.626 \cdot 10^{-27+n}}{c^{2}}[\mathrm{erg}]
$$

where, according to Einstein, mo indicates the inertial mass of the considered quantum object.

As the $\operatorname{erg}$ value is expressed in $\left[\mathrm{g} \cdot \mathrm{cm} / \mathrm{s}^{2} \cdot \mathrm{cm}\right]$, that is in $\left[\mathrm{g} \cdot \mathrm{cm}^{2} / \mathrm{s}^{2}\right]$, we have:

that is:

$$
\begin{aligned}
m_{\circ} & =\frac{6.626 \cdot 10^{-27+n}}{c^{2}}\left[\mathrm{~g} \cdot \mathrm{cm}^{2} / \mathrm{s}^{2}\right] \\
m_{\circ} & =\frac{6.626 \cdot 10^{-27+n}}{\left(2.9979 \cdot 10^{10}\right)^{2}} \frac{\mathrm{g} \cdot \mathrm{cm}^{2} / \mathrm{s}^{2}}{\left[\frac{\mathrm{cm}}{\mathrm{s}}\right]^{2}} \\
m_{\circ} & =7.372 \cdot 10^{-48+\mathrm{n}}[\mathrm{g}]
\end{aligned}
$$

and we have:

Let's to consider a gamma photon $(\nu \mathrm{P})$ with a frequency of $10^{20}[\mathrm{c} / \mathrm{s}]$ which represents the value of $n$ of the previous equations:

$$
\begin{array}{r}
m_{o}=7.372 \cdot\left(10^{-48}+10^{20}\right)[\mathrm{g}] \\
m_{o}=7.372 \cdot 10^{-48+20}[\mathrm{~g}] \\
\text { i.e.: } m_{o}=7.372 \cdot 10^{-28}[\mathrm{~g}]
\end{array}
$$

As can be clearly seen from the Eq.(21), the inertial mass of a $\gamma \mathrm{P}$ is completely superimposable to that of an electron. 
These concepts are not in disagreement with what Amendola reported, so "as primordial cosmic inflation may have been induced by a "particle", or rather by a field, called inflatone, so the recent acceleration could be due, instead that to $\Lambda$, to the hidden work of a field / particle called DE or Quintessence (again Aristotle!) or simply: scalar field. Like all fields, it extends and spreads throughout the space and has its own dynamics. Like all particles, DE has a mass too"[49].

It is just what we stating: the particle that should carry the DE, i.e. the DE Particle (DEP), must have a mass, corresponding, in our opinion, right to the dynamic-mass carried out by the considered photon's momentum, instead of its inertial mass, since the photon( $\mathrm{P})$ always runs madly. To this purpose, Fermi writes: "The photon too, as other particles, is a corpuscle, a light's quantum and has a its own momentum $(\boldsymbol{p})$, through which transfers all its energy to the hit particle"[50].

Feynman adds: "Each photon has an energy and a momentum (p)"[39].

In Newtonian Mechanics $\boldsymbol{p}$ is thus represented:

$$
p \vec{r}=m \cdot \vec{v}
$$

where $m$ is the mass and $v$ the velocity of the involved particle[51].

In Quantum Mechanics, in its turn, $\boldsymbol{p}$ is described by the formula:

$$
p=\frac{h}{\lambda}
$$

where $h$ is the Planck's constant and $\lambda$ is the wavelength of the considered quantum object.

As known, in fact, de Broglie suggested, without experimental data, to give particles the same property as waves. He gave each particle a its own wave length depending only on the momentum $(\boldsymbol{p})$ of the particle itself[52]. According to de Broglie, any quantum object (i.e. any particle) with a momentum $(\boldsymbol{p})$ seems to be something periodic, oscillating as a wave, with an universal relation between the wave length of the particle, indicated by $\lambda$, and modulus $\boldsymbol{p}$ of its momentum [53].

Thus, in line with de Broglie formula, let's now to analyze the $\boldsymbol{p}$ value of photons with different wave length $(\lambda)$. As Weinberg reminds us, the mean wave length of a photon $(P)$ in the optical band corresponds to $\approx 5 \cdot 10^{-5}[\mathrm{~cm}][54]$ and its $p$ is:

$$
\begin{gathered}
p=\frac{h}{\lambda}=\frac{6.626 \cdot 10^{-27}[\mathrm{erg} \cdot \mathrm{s}]}{5 \cdot 10^{-5}[\mathrm{~cm}]} \\
\boldsymbol{P}=\frac{6.626 \cdot 10^{-27}\left[\mathrm{~g} \frac{\mathrm{cm}^{2}}{\mathrm{~s}}\right]}{5 \cdot 10^{-5}[\mathrm{~cm}]} \\
\boldsymbol{p}=1.325 \cdot 10^{-22}\left[\mathrm{~g} \cdot \frac{\mathrm{cm}}{\mathrm{s}}\right]
\end{gathered}
$$

As Eq.(26) shows, the momentum ( $\boldsymbol{p}$ ) of a visible photon carries out a dynamic-mass, a pushing momentum bigger than the rest mass of 100 protons. No surprise! At this regard, Feynman writes: "The momentum, as a mechanical quantity, is difficult to hide. Nevertheless, momentum can be hidden -in the electro-magnetic field, for example. This case is another effect of relativity"[55].

It's like saying that momentum carries, albeit hidden, a dynamic-mass[46]. 
Antonio PUCCINI; Asymptotic Freedom: A Possible Physical Explanation, Advances in Image and Video Processing, Volume 7 No 6, December (2019); pp: 17-40

Feynman states: "In the Einstein Relativity Theory, anything which has energy has mass-mass in the sense that it is attracted gravitationally. Even light, which has en energy, has a mass[39].In fact, Einstein writes to his friend Conrad Habicht: "It has come to my mind a consequence of the study of Electrodynamics. The Principle of Relativity, in association with Maxwell fundamental equations, requires that the mass is a direct measure of the energy contained in a body; the light carries a mass" [56]. In this regard, Galison adds:" Einstein was unsatisfied: he was not satisfied of the analyses of the light. Einstein stated that to any kind of energy is associated a mass" [56].

Thus, according to Einstein there should be a mass associated to the photon(P). Galison continues: "Planck stated that also the transfer of heat adds a mass"[56]. What is heat made of?

As we all know it is made of EMR, that is Ps. Thus, according to Planck, a transfer of radiation, of Ps, from A to B will cause an increase in the mass of B. "It seemed that a hot pot was heavier than a cold one, although exactly the same size. It was a new idea: in Newtonian physics there was nothing suggesting a variation in mass as a consequence of the energy"[56]. Thus wherever there is a body, or particle, having energy, there should be in a way (visible or hidden, concealed) a certain mass too, and vice versa: this is what comes from Eq.(14).

Einstein adds that based on the calculations of its article containing precisely the Eq.(14), it emerges that a body that emits $\mathrm{EMR}_{\mathrm{s}}$ necessarily loses mass.

Feynman goes on: "When a light beam, which has energy in it, comes past the sun there is an attraction on it by the sun. Thus the light does not go straight, but is deflected"[39Vol1]. To this purpose, it is interesting what Eddington announced in 1919: "The simplest interpretation of the deflection of the light beam is the one that considers it as an effect of the weight of light" [57]. At the dinner of that meeting, Eddington read out some verses he had composed; we will quote the last quartine: "We will compare the measures taken, One thing at least is certain, light has weight. One thing is certain and the rest debate. Light rays, when near the Sun, do not go straight"[57].

Thus, Lord Eddington clearly points out the mechanical effect exerted by light, fully in accordance with our conviction that light carries with it also a mass (the dynamic-mass of P). In this respect, Zeilinger chases: "What is the deep meaning of a relationship like $E=m c^{2}$ ? What is hidden behind these symbols? For many physicists the equation $E=m c^{2}$ is to say that energy and mass are the same thing, two faces of the same medal; there is, therefore, equivalence between mass and energy: energy is just another form of mass, and vice versa, mass is another form of energy" [58].

Let's come now to short and very short distances which, we believe, the DE, or Photonic CounterPressure, should operate too.

\subsubsection{Intra-Atomic Spaces}

In agreement with Randall and Barrow, just to mention a few of authors, another operating context of the DE is the intra-atomic space. Randall writes: "As for the world of the atom, probably the most amazing thing is that the atom essentially consists of empty space. The atomic nucleus has a radius of more than 4 orders of magnitude smaller than that of the electronic orbits. The volume of the nucleus is $\approx 10^{\text {?12 }}$ of the volume of the whole atom. An atom is mostly empty, but within this vacuum there is of course an electromagnetic field(EMF), although virtually no real matter is present"[28], but there is energy: the so-called vacuum energy, which is none other than DE which, according to Randall, is actually represented by the $P_{s}$ continuously exchanged between electrons and nucleus. Randall's statement awarded, among other things, of the Honorary Citizenship of Padova, 
just as Hawking, Weinberg and Witten[28], can provide an authoritative and winning asset to our hypothesis.

Along with Randall, Feynman points out: "An EMF has waves, which we call light; it turns out that light also carries a momentum $(\boldsymbol{p})$ with it, so when light impinges on an object it carries in a certain amount of $\boldsymbol{p}$ per second; this is equivalent to a force, because if the illuminated object is picking up a certain amount of $\boldsymbol{p}$ per second, its $\boldsymbol{p}$ is changing and the situation is exactly the same as if there were a force on it. Light makes a pressure when it collides with an objects. It is a very small pressure, but it can be measured with extremely sensitive instruments"[39].

This phenomenon, as known, is interpreted as an "energetic" phenomenon of the $\mathrm{Ps}_{\mathrm{s}}$ (it would be only energy without mass). We are talking about a pressure action, so it should not be unreal to think it is something "real", material, concrete, to produce the pressure effect. Even though it was energy it could be the equivalent mass of the energy to determine the mass effect which hides behind the "photonic pressure". It has been reckoned that the pressure solar rays have on Earth is $1 \mathrm{mg} / \mathrm{mt}^{2}$.

The effect of this pressure induced by solar rays, known as "solar wind", can be observed in the cosmos, when this "wind" gives an impulse to the surfaces it hits. That is, in these cases the intimate light mechanism happens through a "push effect" on electrons. This push effect should be interpreted as a real mechanic effect, rather than energetic.

Then, it is incontrovertible that the EMR exerts a compressive action on the hit object: the so-called Radiation Pressure. It was first pointed out by lohanne Keplero in 1619 this concept of Radiation Pressure just to explain the observation that a tail of a comet always points away from the Sun[59]. Briefly, other than photons $\left(P_{s}\right)$ massless! It is the opposite: with these masses carried out by $P_{s}$ we can better understand, and justify the light pressure action or 'photonic pressure' or radiant pressure [60].

We believe, in fact, that the so-called vacuum energy, that is $\mathrm{DE}$, is nothing transcendental and mysterious: nothing but a form of Photonic Pressure, namely a Photonic counter-Pressure, and the particle that carries this DE most likely is the photon(P).

\subsubsection{Intra-Nuclear Spaces}

Other sites where emerges the action of a repulsive force $(R f)$, having all the charateristics and operational modalities of the ubiquitous $D E$, are represented by the intra-nuclear spaces.

According to Levy, Eisenbud, Wigner and Pacini, in fact, this $R f$ is likely represented by the Levy Interaction, and/or by the barrier mentioned by Pacini. In this respect, it is an EMR barrier, in our opinion, which represents the DE, which is Radiative counter-Pressure.

In other words, we believe that this barrier consists of a multitude of $\mathrm{P}_{\mathrm{s}}$ thickened and crammed together, but without exceeding the limit of the 'compressibility of the radiation'. Although the Ps are bosons, in effect in extreme conditions of pressure and concentrations they are no longer subject to the Pauli Exclusion Principle (PEP)[61].

In this respect, let's analyze with Feynman, one of the most expert in the secrets of light, the Compressibility of the EMR: "We may give one example of the kinetic theory of a gas, one which is not used in chemistry so much, but is used in astronomy. We have a large number of photons in a box in which the temperature is very high. The box is, of course, the gas in a very hot star. The sun is not hot enough; there are still many atoms, but at still higher temperatures in certain very hot stars, we may neglect the atoms and suppose that the only objects that we have in the box are Ps. Now then, a photon has a certain momentum $\boldsymbol{p}$, which is a vector. This $\boldsymbol{p}$ is the $\mathrm{x}$-component of the vector $\boldsymbol{p}$ which generates 
Antonio PUCCINI; Asymptotic Freedom: A Possible Physical Explanation, Advances in Image and Video Processing, Volume 7 No 6, December (2019); pp: 17-40

the kick, and twice the $\mathrm{x}$-component of the vector $\boldsymbol{p}\left(2 p_{\mathrm{x}}\right)$ is the momentum which is given in the kick. Thus we find that the Pressure $(P)$ is:

$$
P=2 n p_{x} v \times
$$

where $n$ is the number of atoms in the volume $V$, and $v$ indicates the number of collisions, that is $n=N / V$ ( $N$ is the total number of atoms). Then, in the averaging, it becomes $\mathrm{n}$ times the average of $p_{\times} v \times$ (the same factor of 2) and, finally, putting in the other two directions, we find:

$$
P V=N \frac{p \cdot v}{3}
$$

That is the pressure times the volume is the total number of atoms times $1 / 3(\boldsymbol{p} \cdot \mathbf{v})$, averaged.

Now, for photons, what is $\boldsymbol{p} \cdot \mathbf{v}$ ? The momentum $(\boldsymbol{p})$ and the velocity $(\mathbf{v})$ are in the same directions, and $\mathbf{v}$ is the speed of light, so this is the momentum of each of the object, times the speed of light. The momentum times the speed of light of every photon is its energy $(E): E=p c$, so these terms are the energies of each of the photons, and we should, of course, take an average energy, times the numbers of photons. So we have $1 / 3$ of the energy inside the gas:

$$
P V=U / 3 \text { (photon gas) }
$$

where $U$ is the total energy of a monoatomic gas. $U$ is equal to a number of atoms times the average kinetic energy of each. So we have discovered that the radiation in a box obeys to the law:

$$
P V^{4 / 3}=C
$$

(where $V$ is the volume and $P$ is the Pressure of the photonic gas). So we know the Compressibility

$(C)$ of the radiation! That is what is used in an analysis of the contribution of radiation pressure in a star, that is how we calculate it, and how it changes when we compress it"[39].

At this point, we must make a reflection: the latter equation gives us a limit, beyond which the radiation cannot be further compressed. And why? Radiation is energy, let's say it is ethereal, it is made up entirely of $P_{s}$, ie massless particles. Furthermore, like all bosons, there can be a large number of $P_{s}$, even in a very limited space, since the PEP does not act on bosons. So Eq.(30) should have almost no limit at all. Instead it is not so: but then, what's underneath? For us, the photonic mass places a limit on the Compressibility of radiation: it is a manifestation of the DE, a sort of $5^{\text {th }}$ Force.

Thus, the fundamental notion of the Compressibility limit of the EMR may help us to understand the possible nature of the barrier mentioned by Pacini[16]. We believe that the secret of the consistency of this barrier, which raises a wall so compact to be able to hold off the intense Strong Interaction (which would inexorably tend to join the nucleons) resides in the even though minuscule mass-energy density conferred to P by the Planck's Constant $(h)$.

Without considering the frequency of the involved $P$, indeed, it still remains its $\boldsymbol{h}$, which as know is not zero, but 6.626? $10^{\text {[27 }}$ [erg? s]. Of course, it is a very small, infinitesimal value. However if we think that in a very small space there can be crammed into billions and billions (the PEP allows it), it is formed over time, under the compressive action, continuous and inexorable of the intra-nucleonic Strong Interaction(SI), or Gluonic Interaction, as a buffer of $P_{s}$ that, in our opinion, becomes progressively more and more incompressible. In this regard, the Eq.(30) shows with extreme precision the limit value of the radiation Compressibility. 
Hence, when density becomes excessive, and the spaces between the particles are extremely reduced, the incomprehensibility of the light and the consistency of $\mathrm{P}_{\mathrm{s}}$ come out.

Consequently the repulsive action takes over, that repulsive, anti-gravitational force represented, governed and managed by Radiation Pressure, as to say by the Photonic counter-Pressure.

However, at this point, one might ask: how is the presence of the Ps justified within the atom? They should be the remitted $P_{s}$ trapped in the 'recombination' phase, which occurred $~ 380^{\circ} 000$ years after the Big Bang [22], when the Ps energy fell to $<13.6 \mathrm{eV}$. A confirmation of this concept is provided by the atomic explosions, which emit in the atmosphere an amazing quantity of visible light, really blinding (whose average energy is $2.48 \mathrm{eV}$ ), in addition to other EMRs.

In our comfort, Randall states: "The intra-atomic space is swarming of Ps"[28].

Basically, with the 'recombination', that is with the formation of atoms, probably a large number of $P_{s}$ are incorporated too, no longer able to break the link between the electron and the proton in a hydrogen atom (whose binding energy is $13.6 \mathrm{eV}$ ).

Therefore, this repulsive force $(R f)$ that acts within the atom, already signaled by Levy, could represent and show probably another mode of action, and of operational place, of DE.

Hence, in this different modus operandi, the DE carries out its action conveyed by sufficiently energetic $P_{s}$, thus demonstrating that the energy density of the DE vary according to the context in which it operates

\subsubsection{Intra-Nucleonic Spaces}

What happens inside the atomic and nuclear space, as previously described, can also occur inside a nucleon, that is in the intra-nucleonic space. In effect, regarding the DE, Pacini writes: "This mysterious repulsive antigravity energy, or $5^{\text {th }}$ Force, should also act against the gluons, thus succeeding in overriding the SI when the $Q_{s}$ tend to get too close to each other, that is when they almost touch each other, but not really: that is there is always some space between the $Q_{s .}$ The space is apparently empty, but actually it is occupied by the 'thickness' of the $5^{\text {th }}$ Force"[16].

We think that the thickness is represented by a large number of $\mathrm{P}_{\mathrm{s}}$, probably too crowded each other, crushed by the Qs in progressive approach (by the SI or gluon force).

This thickness of the repulsive force, probably interposed between $Q_{s}$, may represent just the physical substrate responsible for the peculiar asymptotic freedom phenomenon. In the end, hence, $Q_{s}$ can no longer be compressed further and can no longer be in an increasingly narrow space. This is in disagreement with the PEP, according to which all the bosons can thicken in infinite quantities. At least for the $\mathrm{P}_{\mathrm{s}}$, we must think that there is a limit for the PEP, a limit imposed by the Eq.(30).

In this context, the presence of the $\mathrm{Ps}$, even within the nucleons, dates back to the primordial nucleosynthesis, which started 3 minutes and 46 seconds after the Big Bang [54]. In fact, with this process, many highly energetic $P_{s}$ were trapped inside the nucleons. The demonstration of what we support is provided, this time, by the nuclear explosions, which free a lot of light, similar to the atomic explosions, as well as an abundant emission of highly energetic radiation.

\section{Conclusions}

Summing, when the distance between the $Q_{s}$ is too small, for us may be the thickness of the force (a sort of $5^{\text {th }}$ Force) interposed between the $Q_{s}[18]$ to act as a buffer, triggering, like a spring, (therefore we talk about the DE also as an elastic force) a repulsive action, of mutual removal of the Qs. In our 
Antonio PUCCINI; Asymptotic Freedom: A Possible Physical Explanation, Advances in Image and Video Processing, Volume 7 No 6, December (2019); pp: 17-40

opinion, this thickness behaves in a similar way to the Pacini's saturation barrier, which very likely is interposed both between nucleons within the intra-nuclear space[16] and between the Qs within the intra-nucleonic space. We believe that this repulsive force, or Quintessence, is represented by a multitude of $P_{s}$ that, crammed into an increasingly narrow space, and not further compressible, begin to exert an expansive counter-pressure.

And 'interesting to note that, in such circumstances, the repulsive action of the $D E$, that is the Photonic counter-Pressure in our opinion, performs those tasks attributed to asymptotic freedom. Furthermore, also from this context we deduce that, without the work and the intervention of the DE, the structure of ordinary matter would not have been as it is, or it would not have been there at all! It is as there was something in the intra-nucleonic space, which reveals the effects of his presence only when the $\mathrm{Q}_{s}$ gather excessively. It is as if this something could not be further compressed among $Q_{s}$ too close together, starting to perform a counter-pressure, like a repulsive force. Moreover, the hypothesis that there may be something else inside the gluonic field is not pure fantasy. As Barrow remind us, the seemingly empty space is full of EMR[27], how to say that the socalled vacuum always contains, and in any case, an electro-magnetic field (EMF), that is a Maxwell's field. To this purpose, Penrose adds: "Maxwell EMF delivers energy. For $E=m^{2}$, it must also have a mass. Maxwell's EMF is therefore also matter! Now we must certainly accept this notion"[62]. It is pleonastic to specify that Maxwell's EMF is constituted and operated by Ps. We also know that the intra-hadronic space is not completely empty, it contains the protonic sea [28]. Maybe in addition to the repulsive photonic barrier, these virtual $\mathrm{Q}_{\mathrm{s}}$ and $\overline{\mathrm{Q}}_{\mathrm{s}}$ (that is to say with a very short life), of which protonic sea swarms, contribute to the repulsive action emerging among $Q_{s}$ too close together, and therefore counterbalancing the SI action for excessively short distances.

It is like saying that the so-called shielding effect is not mediated by immaterial, evanescent particles, but by real quantum objects. If we integrate this phenomenon, the explanation will be more exhaustive and complete, to better understand why the action of gluon (G) weakens almost completely for too short distances between $\mathrm{Q}_{s}$ (despite its very intense strength).

For all the above reasons, we believe that the $D E$ is conveyed by $P_{s}$, also of different energies, engaged in various tasks, sometimes peculiar and/or unusual, whose common denominator is represented by the impossibility of being compressed and thickened beyond a determined limit [63].

As concerns the value of the inertial and dynamic mass of photon(P), we can compare, for example, our calculations with those of Penrose, or Perlmutten's, inherent different contexts.

As regards Penrose, in his masterful volume, "The Road to Reality", He writes: "The mass of P, if not 0, should be $<10^{\text {[20 }}$ electronic masses for good observational motives"[64]. The mass of the electron is $9.1 \cdot 10^{\text {[28 }}$ grams, so if the $P$ is $<10^{\text {[20 }}$ electronic masses, we have: $9.1 \cdot 10^{\text {[28]20 }}$ [g]. Thus, in agreement with Penrose a $\mathrm{P}$ which is not massless must have a mass very close to $<9.1 \cdot 10^{\text {@48 }}[\mathrm{g}]$.

Penrose's calculations, among the greatest living mathematicians, are completely superimposable on ours: $7.372 \cdot 10^{-48}[\mathrm{~g}]$, as shown in Eq.(18). This is of great honour for us and greatly comforts us.

As concerns Perlmutten, the surprising accelerated expansion of the Universe has been attributed to a repulsive, anti-gravity action most likely carried out by a mysterious, elusive form of energy, called dark energy (DE). In this case, along with the calculations of Perlmutten team[20], the energy density of this repulsive force, or $\mathrm{DE}$, is $\sim 10^{-29} \mathrm{~g} / \mathrm{cm}^{3}$. 
Thus, in our opinion, this value should represent the energy density of the intergalactic and interstellar DE Particle. DE Particles(DEP) should correspond to $\mathrm{P}_{\mathrm{s}}$ of different wavelengths which, in this context, should be represented by the cosmic microwave background (CMB). As known[65], the CMB wavelength $(\lambda)$ is $\approx 7.35 \cdot 10^{\llbracket 5}[\mathrm{~cm}]$ : they are microwaves, that is $P_{s}$ with very low energy.

Let's see what the $C M B$ energy-mass density is. However, the particles are mostly in motion so that, in the same way as the $P$, to calculate the density value of the DEP mass-energy, we must analyze its momentum $(\boldsymbol{p}): \boldsymbol{p}=h / \lambda$ (as shown in equation 23 ).

Thus, in those circumstances in which the $D E P$ have a $\lambda$ superimposable to that of the $C M B$, the $\boldsymbol{p}$ of the $D E P$, indicated with $D E P$, will be:

$$
\begin{aligned}
& \qquad D E P_{p}=\frac{h}{\lambda}=\frac{6.626 \cdot 10^{-27}[\mathrm{erg} \cdot \mathrm{s}]}{7.35[\mathrm{~cm}]} \\
& \text { that is: } \quad D E P_{p}=0.9014965 \cdot 10^{-27}\left[\mathrm{~g} \cdot \frac{\mathrm{cm}}{\mathrm{s}}\right] \\
& \text { i.e.: } \quad D E P_{p}=9 \cdot 10^{-28}\left[\mathrm{~g} \cdot \frac{\mathrm{cm}}{\mathrm{s}}\right]
\end{aligned}
$$

This finding could represent an indirect counter-proof that the $D E P$ can coincide with the $P_{\mathrm{s}}$, whose $\lambda$ varies according to the context in which they operate[66].

We have proposed, indeed, that DE may also be present within the nuclear space (there it represents the $N$-N Force or Levi's Interaction[14], as to say the Pacini's saturation barrier[16]) and within the nucleonic space, where the DE may create an incompressible physical barrier that prevents $Q_{s}$ to further get closer, probably representing the physical substrate underlying the peculiar $Q_{s}$ asymptotic freedom phenomenon.

In these last circumstances the energy carried by DEP would be much more intense (compared to the intergalactic $D E$ ), so that the respective $\lambda$ would be much shorter than $C M B$ wavelength.

That is the $\lambda$ of the involved DEP (or $\mathrm{P}$, in agreement with our hypothesis) should vary in line with the considered context.

We find very important emphasize that this inconstancy in the energy-mass density value of the $D E$ (and therefore of the $D E P$ ), consequent to the variability of its $\lambda$, is in perfect harmony with Weinberg's concepts which, to make ends meet with the Anthropic Principle, presupposes that the vacuum energy (or DE) took different values in different domains of the Universe [67].

In closing, the counter pressure triggered by DE most likely represents the most immediate physical and real manifestation of an (auxiliary) force or potential energy that appears on occasion when circumstances require it. That is, contrary to the 4 Fundamental Forces, it is as this potential $5^{\text {th }}$ Force, initially present as vacuum energy, represented essentially by electro-magnetic fields swarming of Ps (along with Barrow, continuously exchanged by electrons and ephemeral positrons, generated by the quantum vacuum [27]) was taking shape, structuring in case of necessity, when the compressive action exerted by the Gravity Interaction(GI) becomes excessive, particularly intense, we could say overwhelming, until a counter-reaction takes place, i.e. the Photonic counter-Pressure.

To trigger this counter-pressure, for us is the mass-energy density of a very compact wall of $\mathrm{P}_{\mathrm{s}}$, no further compressible, compressed up to the limit point dictated by the mathematical formalism expressed in Eq.(30), after which the repulsive action is immediately triggered[63]. 
Antonio PUCCINI; Asymptotic Freedom: A Possible Physical Explanation, Advances in Image and Video Processing, Volume 7 No 6, December (2019); pp: 17-40

It is like saying that, in the end, $P_{s}$ are saved in time: what saves them is the limit to their compressibility, elegantly illustrated by Eq.(30). Digging deep, this mathematical limit in our opinion is closely related to a physical parameter: the real structure of a light quantum, the $P$, whose soul is represented by the energy-mass value of the Planck's constant, $h$. It is a truly infinitesimal value, but multiplied by million and millions of times it can acquire a real physical consistency with related effects that cannot be reset.

In this way, we try to embody the possible physical real structure that could be at the bottom of the peculiar $Q_{s}$ asymptotic freedom phenomenon, already masterfully illustrated, from a mathematical point of view, by Gross, Wilczek[1],[2] and Politzer[3].

Moreover, still the Planck constant (precisely through its infinitesimal mass multiplied by the frequency of the $P$ to which it belongs) may represent the mathematical solution to the Yang-Mills mass gap problem, without resorting to the Renormalization or to the Brout-Englert-Higgs Mechanism, but simply inserting its value in all the equations of Perturbative Calculus, Quantum Fields Theory and Yang-Mills Theories[66].

It is clear, in fact, that if we insert this infinitesimal energy-mass value (however $\neq 0$ ) instead of assless photons $\left(P_{s}\right)$ in all these equations, consequently all divergences, that is all zeroes and nfinities, would suddenly disappear.

Accordingly, the limits imposed by the Spontaneous Symmetry Breaking disappear so that there is no longer any need to deny the mass to the Nuclear Forces bosons[68], including the Yang-Mills $\boldsymbol{b}$ quantum[69], i.e. the gluon.

\section{REFERNCES}

[1] Gross D.J., Wilczek F., “Asymptotically Free Gauge Theories. I”, Phys. Rev. D, 8,3633-3652, 1973).

[2] Gross D.J., Wilczek F., "Ultraviolet Behavior of Non-Abelian Gauge Theories",Phys.Rev.Lett., Vol.30, 26, 1343-1346 (1973).

[3] Politzer H.D., "Reliable Perturbative Results for Strong Interactions?”, Phys. Rev. Lett., 30, 1346-1349 (1973).

[4] Maiani L. "60 anni di Yang-Mills", Il Nuovo Saggiatore, 31,5-6, 22-32 (2015).

[5] Bjorken J.D., “Asymptotic Sum Rules at Infinite Momentum”, Phys. Rev.,Vol.179, Iss.5, 1547-1553 (1969).

[6] Altarelli G., Parisi G., “Asymptotic freedom in parton language”,Nucl. Phys., B,Vol.126,Iss.2, 298-318 (1977).

[7] Dokshitzer Y.L., "Calculation of structure functions of deep-inelastic scattering and $\mathrm{e}^{+} \mathrm{e}^{-}$ annihilation by perturbation theory in quantum chromodynamics",Sov.Phys.,JETP,46,4, 641 (1977).

[8] Quigg, C., "Particelle elementari e forze", Le Scienze, Milano (Italian edition of Scientific 
American inc., New York), 2, 20-31 (1985).

[9] Fritzsch H. and Gell-Mann M., "Current Algebra: Quarks and What Else?", Proceedings of the XVI International Conference on High Energy Physics, Chicago,135-165 (1972).

[10] Georgi H., Politzer H.D., "Electroproduction scaling in an asymptotically free theory of strong interactions", Phys.Rev.D,Vol.9, 2, 416-420 (1974).

[11] Gross D.J., Wilczek F., “Asymptotically Free Gauge Theories.II",Phys.Rev. D,Vol.9,4,980-993, (1974).

[12] Appelquist T., Politzer H.D., "Heavy Quarks and $\mathrm{e}^{+} \mathrm{e}^{-}$Annihilation”,Phys.Rev.Lett., Vol.34,1, 43-45 (1975).

[13] Dionisi C.,"QCD", Corso di Fisica Nucleare e Subnucleare II, Roma, INFN, Lesson 5/2/2005.

[14] Levy M.M., "Meson Theory of Nuclear Forces and Low Energy Properties of the NeutronProton System", Phys.Rev.,88,725-738 (1952).

[15] Eisenbud L. and Wigner E.P., "Nuclear Structure", Princeton University Press, N.J.,1958; Boringhieri, Edizioni Universitarie, (Torino),40-41 (1960).

[16] Pacini F., "L’Universo", Editori Riuniti,Roma,158,280-289 (1982).

[17] Heisenberg W.,"Über den Bau der Atomkerne. I”, Zeitschrift für Physik, 77, 1-11 (1932).

[18] http:/www.quibo.it/atosi/quark/confin.htp

[19] Rees A. et al. "Observational Evidence from Supernovae for an Accelerating Universe and a Cosmological Constant", The Astronomical Journal,116,3,1009-1038 (1998).

[20] Perlmutten S. et al., "Measurements of Omega and Lambda from 42 High-Redshift Supernovae", The Astrophysical Journal , 517, 2, 565-586 (1999).

[21] Aczel A. D., “God's Equation”, CAmir D. Aczel 1999,Four Walls Eight Windows ed., New York; il Saggiatore ed., Milano, 25,138,141,175 (2000).

[22] Hack M., “Dove nascono le stelle”, Sperling \& Kupfer ed.,Milano,164-166,169-171 (2004).

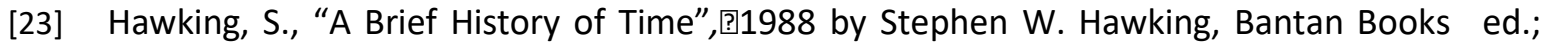
Rizzoli ed.,Milano,126 (1990).

[24] Chandrasekhar B.S., "Why Things Are the Way They Are", Cambridge University Press,1998; II Saggiatore ed., Milano, 84,125,304 (2004).

[25] Puccini A., "About the Zero Point Energy, Zero Point Mass, Zero Point Temperature and Zero Point Motion in the Subatomic World and Photonics", Proceedings of Progress in Electromagnetics Research Symposium, Suzhou, China, 1169-1171,Sept. 12-16 (2011).

[26] Ferris T., "Matter and Dark Energy", National Geographic, Italian edition,1,74-89 (2015). 
Antonio PUCCINI; Asymptotic Freedom: A Possible Physical Explanation, Advances in Image and Video Processing, Volume 7 No 6, December (2019); pp: 17-40

[27] Barrow J.D., "The Book of Nothing", (C) John D. Barrow 2000,1st published by Jonathan Cape 2000; Mondadori Ed., Milano,220-221,239-240 (2001).

[28] Randall L., “Knocking on Heaven's Door”, CLisa Randall 2011-2012; il Saggiatore ed., (Milano),92,93,442,381,98 (2012).

[29] Bhattacharjee Y., "The Universe”, National Geographic, Italian edition,4,42-67 (2014).

[30] Dark matter | CERN | https://home.cern/science/physics/dark-matter

[31] http:///map.gsfc.NASA.m uni/uni 101bb2.3.html

[32] European Space Agency (ESA): Planck and the Cosmic Microwave Background, https://www.esa.int/Our Activities/Space Science/Planck/Planck and the cosmic microw $\underline{\text { ave }}$

[33] The Planck Collaboration, "Planck 2013 results. 1.Overview of products and scientific results", Astronomy \& Astrophysics, Vol.571, A1,1-48, November (2014).

[34] Rovelli C., "Così è rinato l'Universo", il Sole 24 ore, Milano, 276,33 (2011).

[35] Ashtekar A., "Gravity, Geometry and the Quantum", arXiv:gr-qc/0605011v2 14 Jul 2006

[36] Puccini A., "The Inflationist Expansion of the Universe Was Conducted by Very High Energy Photons" (Session 3P1: Optics and Photonics). Progress in Electromagnetics Research Symposium Proceedings, Cambridge (Ma), 819-822, July 5-8 (2010).

[37] Puccini A., "Electron Capture: a More Complete Equation", viXra.org, Mathematical Physics: 1809.0232 (2018).

[38] Puccini A., "Neutronization: a More Comprehensive Equation", International Journal of Current Research, Vol.11, Iss.7, 5805-5812 (2019).

[39] Feynman R.P., "The Feynman Lectures on Physics". Vol.1, 1965,1989 California Institute of Technology (Caltech); Zanichelli Ed., Bologna,vol.1, 34-5,16,17;10-13;17-7;39-8(2001).

[40] Puccini A., "Light Weighs", Progress in Electromagnetics Research B, Vol.8,161-177 (2008).

[41] Nat'l Academies Press, The National Academy of Science, USA, "Nuclear Physics. Chapter 5: Nuclear Astrophysics",111(1986).

[42] Puccini A., "The Equivalent Rest-mass of Photon" (Session 2P2: Electromagnetic Theory and Design on the Optical Dispersive Materials, Invisible Cloak and Photonic Crystals). Proceedings of Progress in Electromagnetics Research Symposium, Marrakesh, Morocco,723-725, Mar. 20-23 (2011).

[43] Puccini A., "About the Zero Mass of Photon", Progress in Electromagnetics Research, (PIER), Vol.55,117-146 (2005).

[44] Puccini A., "Quantum Gravity is Induced by a Mechanical Effect Elicited by Momentum of 
Light's Quanta", Asian Journal of Science and Technology,Vol.10,1,9206-9220 (2019).

[45] Puccini A..,"A Mechanical Effect Induced by Electromagnetic Radiation May Explain the Wave Function Collapse of a Quantum Object" (Session 2P2: Electromagnetic Theory and Design on

[46] the Optical Dispersive Materials, Invisible Cloak and Photonic Crystals). Proceedings of Progress in Electromagnetics Research Symposium, Marrakesh, Morocco,726-728, Mar. 2023 (2011).

[47] Puccini A., "Light Quantum Induces the Measurement Paradox", Journal of Advances in Physics, Vol.15, 6039-6055 (2018).

[48] Guth A., "Inflationary universe: A possible solution to the horizon and flatness problems", Physical Review D, Vol.23,N²,347-356 (1981).

[49] Einstein A., "Ist die Tragheit eines Korpers von seinem Energieinhalt abhangig?", Annalen der Physik, Vol.18,639-641 (1905).

[50] Amendola L., "Costante Cosmologica, Energia Oscura ed Espansione dell'Universo", INFN, http:/www.asimmetrie.it/tags/tag/energia-oscura

[51] Fermi E.(1926), "Argomenti pro e control'ipotesi dei quanti di luce", Nuovo Cimento,3,201206.

[52] Newton Is.(1687), "PHILOSOPHIAE NATURALIS PRINCIPIA MATHEMATICA". Imprimatur S.Pepys, Reg.Soc. Praeses. iulii 5.1686.Londini. Anno MDCLXXXVII

[53] de Broglie L., “Ondes et quanta”, Comtes Rendus, Vol.177, 507-510 (1923).

[54] Puccini A., "The Momentum of Photon May Explain the Measurement's Paradox in the Subatomic World" (Session 1A2: Electromagnetic Theory and Design on the Optical Dispersive Materials, Invisible Cloak and Photonic Crystals). Proceedings of Progress In Electromagnetics Research Symposium, Suzhou, China,27-30,Sept.12-16 (2011).

[55] Weinberg S., "The First Three Minutes. A Modern View of the Origin of the Universe", (c 1977 by S.Weinberg; Mondadori Ed.(Milano),75,125 (1977).

[56] Feynman R.P., The Feynman Lectures on Physics, Vol.3: Quantum Mechanics, (1965, California Institute of Technology; Zanichelli Ed., Bologna,Vol.3,2-4 (2001).

[57] Galison P., "The Sextant Equation: $E=m . c^{2 " ~ i n ~ " I t ~ M u s t ~ b e ~ B e a u t i f u l . ~ G r e a t ~ e q u a t i o n s ~ o f ~}$ Moderne Science"; Granta Publ.,London,2002; il Saggiatore ed.,Milano,119 (2005).

[58] Eddington A., "Joint Eclipse Meeting of the Royal Society and the Royal Astronomical Society", in The Observatory (Review of Astronomy),vol.27, 545,389,Nov.(1919).

[59] Zeilinger A.,"Einsteins Schleier. Die neve Welt der Quantenphysik", 2003,Verlag C.H., Munchen; G. Einaudi ed.,Torino,130-131 (2005).

[60] Keplero Johanne, "De Cometis Libelli Tres."; publisher Augustae Vindelicorum, Andrea 
Aspergeri, Auguftani (1619).

[61] Puccini A., "The Missing Mass in the Universe May Be Represented by the Dynamic-mass of the Photons" (Session 3P1: Optics and Photonics). Proceedings of Progress in Electromagnetics Research Symposium, Cambridge (Ma), 816-818, July 5-8 (2010).

[62] Pauli W., "Über den Zusammenhang des Abschlusses der Elektronengruppen im Atom mit der Komplexstruktur der Spektren", Zeitschrift für Physik, 31, 765-783 (1925).

[63] Penrose R., "The Emperor's New Mind”, (01989 Oxford Univerity Press; Rizzoli (RCS) Ed., Milano, 287 (1997).

[64] Puccini A., "Dark Energy Particle is the Photon", viXra.org, Astrophisics:1903.0359 (2019).

[65] Penrose R., "The Road to Reality", 2004 Roger Penrose; 2005,RCS ed., Milano, 641 (2005).

[66] Penzias A.A., Wilson R.W.,"A Measurement of Excess Antenna Temperature at $4080 \mathrm{Mc} / \mathrm{s}^{\text {", }}$ Astrophysical Journal,142,419-421 (1965).

[67] Puccini A., "Dark Energy Particles May Be Photons", International Journal of Science and Research,Vol.8, Iss.8, 473-491 (2019).

[68] Weinberg S., "Anthropic Bound on the Cosmological Constant", Phys. Rev.Lett., 59, 22, 26072610 (1987).

[69] Puccini A., "On the Bosons' Range of the Weak Interaction", Journal of Advances in Physics, Vol.14, Issue 3, 5865-5868 (2018)

[70] Yang C.N. and Mills R.L., "Conservation of Isotopic Spin and Isotopic Gauge Invariance”, Phys. Rev., 96,1,191-195 (1954). 